\title{
HARMONIC ANALYSIS ON NILMANIFOLDS
}

\author{
BY \\ JONATHAN BREZIN
}

\begin{abstract}
We compute, using a device of A. Weil, an explicit decomposition of $L^{2}$ of a nilmanifold into irreducible translation-invariant subspaces. The results refine previous work of C. C. Moore and L. Green.
\end{abstract}

1. Introduction. Let $N$ be a connected and simply connected nilpotent Lie group that contains a discrete, cocompact subgroup $\Gamma$. On the compact manifold $N / \Gamma$ there is a measure $\mu$ which is invariant under translation by elements of $N$. Thus translation by an element $n$ of $N$ defines a unitary operator $U_{n}^{\Gamma}$ on $L^{2}(N / \Gamma, \mu)$ $=\boldsymbol{H}$ as

$$
\left(U_{n}^{\Gamma} f\right)(\Gamma m)=f(\Gamma m n)
$$

for all $m$ and $n$ in $N$. The map $n \mapsto U_{n}^{\Gamma}$ defined a unitary representation $U^{\Gamma}$ of $N$ on $\boldsymbol{H}$. It is known that $\boldsymbol{H}$ decomposes into discrete direct sum of irreducible in- variant subspaces for $U^{\Gamma}$. Indeed, under a mild condition on $\Gamma$, Calvin C. Moore has computed explicitly which irreducible unitary representations of $N$ occur in $U^{\Gamma}$. Moore's result is quoted in the next section. The object of this paper is to use Moore's result to obtain an explicit direct-sum decomposition of $\boldsymbol{H}$ into irreducible invariant subspaces.

The argument we shall use was motivated by a construction used by A. Weil in [6]. Our variant of Weil's construction applies directly only when $N$ is two-step nilpotent-that is, when $[N, N]$ is central in $N$. We have therefore isolated this case in $\$ \$ 3$ and 4 . The result we have for general $N$ is an algorithm that enables one to reduce the general case to the two-step case.

It is not surprising that we should end up with an algorithm (i.e. and not an intrinsic description of the subspaces). The fact is that although it is known what irreducible unitary representations of $N$ occur in $U^{\Gamma}$, only the crudest of estimates can be made by present techniques of the multiplicities, at least for general $N$. (See Moore's discussion of this problem in [4].) The problem is that both Moore's approach and our approach involve induction on the dimension of $N$, and in the course of this induction, the multiplicities are presented only as the solutions to Diophantine equations of great complexity.

Received by the editors September 24, 1968.

AMS Subject Classifications. Primary 2265.

Key Words and Phrases. Nilpotent Lie group, nilmanifold, unitary representations, theta-functions.

Copyright (C) 1970, American Mathematical Society 
2. Moore's result. We begin by recalling Kirillov's correspondence. Let $\mathfrak{R}$ denote the Lie algebra of $N$, and let $\varphi$ denote a linear functional on $\mathfrak{R}$. A subalgebra $\mathfrak{S}$ of $\mathfrak{N}$ is said to be subordinate to $\varphi$ if $\varphi([\mathfrak{H}, \mathfrak{S}])=0$, or in other words, if the restriction of $\varphi$ to $\mathfrak{F}$ defines a Lie algebra homomorphism from $\mathfrak{S}$ into the real numbers $\boldsymbol{R}$.

Let us assume that we are given a subalgebra $\mathfrak{S}$ of $\mathfrak{N}$ subordinate to $\varphi$, and let $H$ denote the connected subgroup of $N$ corresponding to $\mathfrak{S}$. Because the restriction of $\varphi$ to $\mathfrak{F}$ is a Lie algebra homomorphism, there exists a Lie group homomorphism $\Phi: H \rightarrow \boldsymbol{R}$ whose derivative at the identity of $H$ is the restriction of $\varphi$ to $\mathfrak{F}: d \Phi=\varphi \mid \mathfrak{H}$. The map $h \mapsto \exp (2 \pi i \Phi(h))$ defines a character $\chi$ of $H$. We shall use $I_{N}(\varphi, \mathfrak{S})$ to denote the unitary representation of $N$ induced (in Mackey's sense) by the character $x$.

TheOREM (KIRILlov [3]). (1) If $\mathfrak{S}$ is chosen of maximal dimension among those subalgebras of $\mathfrak{N}$ subordinate to $\varphi$, then $I_{N}(\varphi, \mathfrak{F})$ is irreducible.

(2) If $\mathfrak{S}$ and $\mathfrak{R}$ are any two subalgebras of $\mathfrak{R}$ of maximal dimension among those subordinate to $\varphi$, then $I_{N}(\varphi, \mathfrak{S})=I_{N}(\varphi, \mathfrak{R})$.

Let $\mathfrak{R}^{*}$ denote the space of all linear functionals on $\mathfrak{N}$. We shall use $I_{N}$ to denote the map which assigns to an element $\varphi \in \mathfrak{R}^{*}$ the unitary equivalence class $I_{N}(\varphi, \mathfrak{S})$ described in (2).

(3) $I_{N}$ maps $\mathfrak{N}^{*}$ onto the space $\hat{N}$ of all unitary equivalence classes of irreducible unitary representations of $N$. Furthermore, if $\varphi, \psi \in \mathfrak{N}^{*}$, then $I_{N}(\varphi)=I_{N}(\psi)$ if, and only if, there is some $n \in N$ such that $\varphi(x)=\psi(\operatorname{Ad}(n) x)$ for all $x \in \mathfrak{R}$.

$I_{N}$ is called Kirillov's correspondence.

Crucial to Moore's application of Kirillov's theorem is the following notion:

Definition. Recall that, $N$ being nilpotent, the exponential map exp: $\mathfrak{R} \rightarrow N$ is a homeomorphism. Let log denote the inverse of exp. We shall call the discrete, cocompact subgroup $\Gamma$ of $N$ a lattice subgroup if, and only if, $\log \Gamma$ is a subgroup of the underlying additive group of the Lie algebra $\mathfrak{N}$ of $N$.

REMARKS. The notion of lattice subgroups is useful in many situations other than that we are about to consider. See [1], for example. Moore's paper contains a thorough discussion of the existence of lattice subgroups.

Theorem (C. C. Moore [4]). Let $\Gamma$ be a lattice subgroup of $N$ and let $\varphi$ be a linear functional on the Lie algebra $\mathfrak{R}$ of $N$. Then $I_{N}(\varphi)$ occurs in $U^{\Gamma}$ if, and only if, there is some $n \in N$ such that $\varphi \circ \operatorname{Ad}(n)$ maps $\log (\Gamma)$ into the integers $Z$.

For previous work in this direction, see L. Green's work in [2].

3. A reduction lemma. In addition to the assumptions already made on $N$ and $\Gamma$ (in the introduction), we now impose the assumptions that

(1) $N$ is two-step nilpotent.

(2) $\Gamma$ is a lattice subgroup of $N$. 
We remark that conditions for the existence of such pairs $(N, \Gamma)$ are considered in detail by J. Scheuneman in [5].

Let $\varphi$ be a linear functional on $\mathfrak{R}$ that maps $\log (\Gamma)$ into $Z$. By Moore's theorem $I_{N}(\varphi)$ is a subrepresentation of $U^{\Gamma}$. The Hilbert space $\boldsymbol{H}$ of $U^{\Gamma}$ can be decomposed into a direct sum $\boldsymbol{K}(\varphi) \oplus \boldsymbol{L}(\varphi)$ in such a way that $\boldsymbol{K}(\varphi)$ and $\boldsymbol{L}(\varphi)$ are both invariant under $U^{\Gamma}$, the restriction of $U^{\Gamma}$ to $K(\varphi)$ is equivalent to a finite multiple of $I_{N}(\varphi)$, and $I_{N}(\varphi)$ is not a subrepresentation of the restriction of $U^{\Gamma}$ to $L(\varphi)$. Our immediate objective is to compute $\boldsymbol{K}(\varphi)$.

Let $\Re$ be the kernel of $\varphi$, and let $\Re$ be the set of all $x$ in $\Re$ satisfying $\varphi([x, \Re])=0$. Since $[\mathfrak{R}, \mathfrak{R}]$ is central in $\mathfrak{R}$, we have $[\mathfrak{R}, \mathfrak{R}] \subseteq \Re$, and hence $\mathfrak{R}$ is an ideal in $\mathfrak{R}$. Because $[\Re, \Re] \subseteq \Re \cap \Re, \Re \cap \Re$ is also an ideal in $\mathfrak{R}$.

There are two possibilities to consider:

The first possibility is that $[\mathfrak{R}, \mathfrak{\Re}] \subseteq \Re$. When that happens, $I_{N}(\varphi)$ is a character of $N$, and $\boldsymbol{K}(\varphi)$ is the space of all $f$ in $\boldsymbol{H}$ satisfying

$$
f(\Gamma m n)=\left[\left(I_{N}(\varphi)\right)(n)\right] f(\Gamma m)
$$

for all $m$ and $n$ in $N$.

The remaining possibility is that $[\mathfrak{R}, \mathfrak{R}] \nsubseteq \Re$. Here matters are more complicated. We begin with a lemma:

Let $A=\exp (\Re \cap \Re) . A$ is then a closed, normal subgroup of $N$.

LEMMA. $A \Gamma$ is a closed subgroup of $N$.

Proof. We shall prove that $A \Gamma=\exp (\log (\Gamma)+(\Re \cap \Re))$ and that $\log (\Gamma)$ $+(\Re \cap \Re)$ is a closed subgroup of $\Re$. This will prove the lemma.

The lemma is proved by means of the Campbell-Hausdorff formula, which, in the case at hand, takes the form

$$
\exp (x+y) \exp \left(\frac{1}{2}[x, y]\right)=\exp (x) \exp (y),
$$

because $[x, y]$ is central in $\mathfrak{R}$.

If $x$ and $y$ are in $\log (\Gamma)$, then $x+y \in \log (\Gamma)$, and hence by the CampbellHausdorff formula, $\frac{1}{2}[x, y] \in \log (\Gamma)$. We have thus shown that $[\log (\Gamma), \log (\Gamma)]$ $\subseteq \log (\Gamma)$. Thus

$$
[\log (\Gamma)+(\Re \cap \Re), \log (\Gamma)+(\Re \cap \Re)] \subseteq 2(\log (\Gamma)+(\Re \cap \Re)) .
$$

Hence the Campbell-Hausdorff formula implies that $\exp (\log (\Gamma)+(\Re \cap \Re))$ is a subgroup of $N$ and that this subgroup is generated by $\Gamma$ and $A$. In other words, $\exp (\log (\Gamma)+(\Re \cap \Re))=A \Gamma$.

It remains now to show that $\log (\Gamma)+(\Re \cap \Re)$ is a closed subgroup of $\Re$. In order to prove this, we need only prove that $\Re \cap \Re$ has a basis consisting of rational linear combinations of elements from a basis for the free abelian group $\log (\Gamma)$. In other words, we must prove that the rank of $\log (\Gamma) \cap \Re \cap \Re$ as a free abelian group equals the dimension of $\Re \cap \Re$, and this is completely obvious. Q.E.D. 
We now know that $\Gamma A / A$ is a lattice subgroup of $N / A$. Moreover, $A$ lies in the kernel of the representation $I_{N}(\varphi)$, as one can easily see by retracing the construction of $I_{N}(\varphi)$ from $\varphi$. It follows that the subspace $K(\varphi)$ is contained in $\{f \in \boldsymbol{H}: f(\Gamma n a)$ $=f(\Gamma n)$ for all $n \in N$ and $a \in A\}$. Hence, by denoting by $\bar{\varphi}$ the linear functional on $N / R \cap K$ defined by $\varphi$, we see that there is a natural map from $K(\varphi)$ onto the corresponding subspace for $I_{N / A}(\bar{\varphi})$ in $L^{2}(N / A \Gamma, \nu)$, where $\nu$ is an invariant measure on $N / A \Gamma$ normalized so that the map $K(\varphi) \rightarrow L^{2}(N / A \Gamma, \nu)$ is an isometry.

Thus, in order to describe $K(\varphi)$, it is enough to describe the corresponding subspace of $L^{2}(N / A \Gamma, \nu)$. But $N / A$ is a very special sort of group:

Lemma. The commutator subgroup of $N / A$ and the center of $N / A$ are equal and both are one dimensional.

Proof. If $x$ is central in $\mathfrak{R} / \mathfrak{R} \cap \Re$, then $\bar{\varphi}([x, \mathfrak{R}])=0$ and hence $x \in \mathfrak{R} / \mathfrak{R} \cap$. Since $\varphi$ does not vanish on $[\mathfrak{R}, \mathfrak{R}]$, it follows that $[N / A, N / A]=R / A$.

One can easily see that $N / A$ is isomorphic to one of the groups $H(r)$, where $H(r)$, for a positive integer $r$, is the group of all real matrices of the form

$$
\left(\begin{array}{cccccc}
1 & x_{1} & x_{2} & \cdots & x_{r} & z \\
0 & 1 & 0 & \cdots & 0 & y_{1} \\
& 0 & \ddots & & \vdots & y_{2} \\
\vdots & \vdots & & 1 & 0 & \vdots \\
& & & & 1 & y_{r} \\
0 & 0 & \cdots & . & 0 & 1
\end{array} \mid\right.
$$

$H(r)$ is sometimes called the $r$ th Heisenberg group.

4. Solution of the main problem for the Heisenberg groups. We now assume that $N=H(r)$ for some $r$. We shall first compute the possibilities for $\Gamma$. Let $\mathfrak{L}$ denote $\log (\Gamma)$, and let $3=\mathfrak{L} \cap[\mathfrak{R}, \mathfrak{N}] . \mathfrak{L} / \mathbb{Z}$ is torsion-free, since it is a subgroup of $\mathfrak{R} /[\mathfrak{N}, \mathfrak{R}]$. Hence $\mathfrak{L} / \mathbb{Z}$ is free and the extension

$$
0 \rightarrow \mathfrak{B} \rightarrow \mathfrak{L} \rightarrow \mathfrak{L} / \mathbb{B} \rightarrow 0
$$

splits. Hence $\mathfrak{L}=\mathfrak{B} \oplus \mathbb{Z}$ for some $\mathfrak{B}$. As we saw above, $[\mathfrak{L}, \mathfrak{L}] \subseteq \mathbb{Z}$-in fact, $[\mathfrak{L}, \mathfrak{Q}] \subseteq 28$. Thus $[\cdot, \cdot]$ is an alternating, nondegenerate bilinear form on $V$.

Choose an element $z$ in 3 that generates 3 . Because $[\cdot, \cdot]$ is alternating and nondegenerate on $\mathfrak{B}$, we can choose a basis $x_{1}, \ldots, x_{r}, y_{1}, \ldots, y_{r}$ for $\mathfrak{B}$ with the following properties:

(a) $\left[x_{i}, x_{j}\right]=\left[y_{i}, y_{j}\right]=0$, and

(b) $\left[x_{i}, y_{j}\right]=a_{i} \delta_{i j} z$

for $1 \leqq i, j \leqq r$. Set $u_{i}=a_{i}^{-1} y_{i}$. Then $\left\{x_{1}, x_{2}, \ldots, x_{r}, u_{1}, \ldots, u_{r}, z\right\}$ is a basis for $\mathfrak{R}$ with the familiar multiplication table $\left[x_{i}, u_{j}\right]=\delta_{i j} z$.

Let $\mathfrak{N}^{*}$ be the space of all linear functionals on $\mathfrak{N}$, and let $\left\{x_{1}^{*}, \ldots, x_{r}^{*}, u_{1}^{*}, \ldots\right.$, $\left.u_{r}^{*}, z^{*}\right\}$ be the basis for $\mathfrak{R}^{*}$ dual to the given basis of $\mathfrak{R}$. Then $\varphi=m_{1} x_{1}^{*}+m_{2} x_{2}^{*}$ 
$+\cdots+m_{r} x_{r}^{*}+n_{1} u_{1}^{*}+\cdots+n_{r} u_{r}^{*}+p z^{*}$, where $m_{i} \in \boldsymbol{Z}, a_{i} n_{i} \in \boldsymbol{Z}$, and $p \in \boldsymbol{Z}$, and $p \neq 0$. Furthermore, one can easily see from Kirillov's theorem that $I_{N}(\varphi)$ is uniquely determined by $p=\varphi(z)$, and also does not depend on $m_{\mathfrak{i}}$ or $n_{\mathfrak{i}}$.

If we take for the maximal subordinate subalgebra the subalgebra generated by $u_{1}, \ldots, u_{r}$ and $z$, then $I_{N}(\varphi)$ can be realized on

$$
L^{2}\left(\boldsymbol{R} x_{1} \times \boldsymbol{R} x_{2} \times \cdots \times \boldsymbol{R} x_{r}, d x_{1} d x_{2} \cdots d x_{r}\right)
$$

in the following manner:

Let $\chi=\sum_{i=1}^{r} \chi_{i} x_{i}, \eta=\sum_{i=1}^{r} \eta_{i} x_{i}$, and $\zeta \in \boldsymbol{R}$. Denote by $\langle\chi, \eta, \zeta\rangle$ the group element $\exp \left(\chi+\eta+\left(\zeta-\frac{1}{2} \chi \cdot \eta\right) z\right)$, where $\chi \cdot \eta=\sum_{i=1}^{r} \chi_{i} \eta_{i}$. Then define

$$
\left(U_{\langle x, \eta, \zeta\rangle}^{\varphi} f\right)(\lambda)=[\exp 2 \pi i(p(\zeta+\lambda \cdot \eta)+n \cdot \eta)] f(\lambda+\chi)
$$

for all $\lambda=\sum_{i=1}^{r} \lambda_{i} x_{i}$ and all $f \in L^{2}\left(R^{r}\right)$, where $n \cdot \eta$ is defined to be $\sum_{i=1}^{r} n_{i} \eta_{i}$. Using the group law in $N$ in the form

$$
\langle\chi, \eta, \zeta\rangle\langle\rho, \sigma, \tau\rangle=\langle\chi+\rho, \sigma+\eta, \zeta+\tau+\chi \cdot \sigma\rangle,
$$

one can easily verify that $U^{\varphi}$ is a homomorphism. $U^{\varphi}$ is a unitary representation of $N$ whose unitary equivalence class is $I_{N}(\varphi)$.

We shall now exhibit an operator $V^{\varphi}$ that carries $L^{2}\left(R^{r}\right)$ into $L^{2}(N / \Gamma, \mu)$ in such a way that (1) $V^{\varphi}$ is an isometry if $\mu$ is appropriately normalized and $(2) V^{\varphi} U^{\varphi}\left(V^{\varphi}\right)^{-1}$ is the restriction of $U^{\Gamma}$ to $V^{\varphi}\left(L^{2}(R)\right)$. We remark that the operator $V^{\varphi}$ we are about to define is a mild variant of an operator introduced in a similar context by A. Weil in [6]; see p. 164 et seq in [6].

Let $f$ be a $C^{\infty}$-function on $\boldsymbol{R}^{r}$ with compact support. Define

$$
\left(V^{\varphi} f\right)(\langle\chi, \eta, \zeta\rangle)=\exp 2 \pi i(p \zeta+\boldsymbol{n} \cdot \eta) \sum_{\boldsymbol{m} \in \mathbf{Z}^{\boldsymbol{r}}} f(\chi+\boldsymbol{m}) \exp 2 \pi i p(\boldsymbol{m} \cdot \eta) .
$$

As we have defined it, $V^{\varphi} f$ is a $C^{\infty}$-function on $N$. We shall now show that $V^{\varphi} f$ is constant on right $\Gamma$ cosets. Let $\langle\boldsymbol{p}, \boldsymbol{q}, \boldsymbol{r}\rangle \in \Gamma$. Recall that $\boldsymbol{p} \in \boldsymbol{Z}^{r}, \boldsymbol{r} \in \boldsymbol{Z}$ and $q=\sum_{i=1}^{r} q_{i} u_{i}$ where $a_{i} \mid q_{i}$. Now

$$
\begin{aligned}
\left(V^{\varphi} f\right)(\langle\boldsymbol{p}, \boldsymbol{q}, \boldsymbol{r}\rangle\langle\chi, \eta, \zeta\rangle) & \\
& =\exp 2 \pi i(p(\zeta+r+\boldsymbol{p} \cdot \eta)+\boldsymbol{n} \cdot(\eta+\boldsymbol{q})) \cdot \sum_{\boldsymbol{m} \in \mathbf{Z}^{\boldsymbol{r}}} f(\chi+\boldsymbol{p}+\boldsymbol{m}) \exp 2 \pi i p(\boldsymbol{m} \cdot(\eta+\boldsymbol{q})) \\
& =\exp 2 \pi i[p(\zeta+\boldsymbol{p} \cdot \eta)+\boldsymbol{n} \cdot \eta] \sum_{\boldsymbol{m}} f(\chi+\boldsymbol{m}) \exp 2 \pi i p((\boldsymbol{m}-\boldsymbol{p}) \cdot \eta) \\
& =\exp 2 \pi i[p \zeta+\boldsymbol{n} \cdot \eta] \sum_{\boldsymbol{m}} f(\chi+\boldsymbol{m}) \exp 2 \pi i p(\boldsymbol{m} \cdot \eta),
\end{aligned}
$$

as desired. Thus we shall view $V^{\varphi} f$ as a function on $N / \Gamma$. As Weil points out in different terms, the fact that $V^{\varphi} f$ is well-defined modulo $\Gamma$ corresponds in the special case $r=1$ for the special function $f(x)=\exp \left(-x^{2}\right)$ to the familiar transformation rules for $\theta$-functions.

For a proof that $V^{\varphi}$ is a constant multiple of an isometry, see Weil's paper, p. 165. 
Let $f$ be as above, and let $\langle\chi, \eta, \zeta\rangle$ and $\langle\rho, \sigma, \tau\rangle \in N$. Then

$$
\begin{aligned}
\left(V^{\oplus} U_{\langle\rho, \sigma, \tau\rangle}^{\varphi} f\right)(\langle\chi, \eta, \zeta\rangle) & \\
\quad & =\exp 2 \pi i(p \zeta+n \eta) \sum_{\boldsymbol{m}}\left(U_{\zeta \rho, \tau, \tau\rangle}^{\varphi} f\right)(\chi+\boldsymbol{m}) \exp 2 \pi i p(\boldsymbol{m} \cdot \eta) \\
& =\exp 2 \pi i(p \zeta+n \eta) \sum_{\boldsymbol{m}} \exp 2 \pi i[p(\tau+(\chi+\boldsymbol{m}) \cdot \sigma)+\boldsymbol{n} \sigma] f(\chi+\rho+\boldsymbol{m}) \exp 2 \pi i p(\boldsymbol{m} \cdot \eta) \\
& =\exp 2 \pi i[p(\zeta+\tau+\chi \cdot \sigma)+\boldsymbol{n}(\eta+\sigma)] \sum_{\boldsymbol{m}} f(\chi+\rho+\boldsymbol{m}) \exp 2 \pi i p \boldsymbol{m} \cdot(\sigma+\eta) \\
& =\left(V^{\varphi} f\right)(\langle\chi, \eta, \zeta\rangle\langle\rho, \sigma, \tau\rangle) \\
& =\left(U_{\langle\rho, \sigma, \tau\rangle}^{\Gamma} V^{\varphi} f\right)(\chi, \eta, \zeta) .
\end{aligned}
$$

We have thus proved that $V^{\varphi} U_{\langle\rho, \sigma, \tau\rangle}^{\varphi}\left(V^{\varphi}\right)^{-1}$ is $U_{\langle\rho, \sigma, \tau\rangle}^{\Gamma}$ restricted to $V^{\varphi}\left(L^{2}\left(R^{r}\right)\right)$.

TheOREM. $K(\varphi)=\sum_{\theta} \oplus V^{\theta}\left(L^{2}\left(R^{r}\right)\right)$, where $\theta$ traces $\left\{\psi \in \mathfrak{L}^{*}: \psi(z)=p\right.$ and $0 \leqq$ $a_{i} \psi\left(u_{i}\right)<p$ for all $\left.i, 1 \leqq i \leqq r\right\}$.

Proof. That the sum is direct is clear from the fact that the defining sums for $V^{\theta}$ involve different $\exp (2 \pi i c \cdot \eta)$ terms for different values of $\theta$. That the sum is exhaustive is equally clear. (Cf. Moore's computation of the multiplicities in the case $r=1-[4$, p. 157].) Q.E.D.

5. The general case. We now consider the case where $\Gamma$ is a lattice subgroup of a simply connected and connected, but otherwise arbitrary nilpotent Lie group $N$. Let $\varphi$ be a linear functional on the Lie algebra $\mathfrak{R}$ of $N$. We assume that $\varphi$ takes integer values on the lattice $\log (\Gamma)$, so that $I_{N}(\varphi)$ occurs in $U^{\Gamma}$. We shall use $\boldsymbol{K}(\varphi) \oplus \boldsymbol{L}(\varphi)$ to denote the direct sum decomposition of $\boldsymbol{H}=L^{2}(N / \Gamma, \mu)$ described at the beginning of $\S 3$, above. Our object is to give an inductive method for computing a direct-sum decomposition of $\boldsymbol{K}(\varphi)$ into irreducible subspaces.

We begin with a reduction analogous to that of $\S 3$. Let $\Re=\{x \in \mathfrak{R}: \varphi(x)=0$ and $[x, \mathfrak{R}]=0\}$. Clearly $\mathfrak{R}$ is of codimension one in the center of $\mathfrak{R}$. One can easily show that the center of $\mathfrak{N}$ has a basis consisting of elements of $\log (\Gamma)$. It then follows from the integrality of $\varphi$ on $\log (\Gamma)$ that such a basis can also be found for $\Re$. Hence $\Gamma \exp (\Re)=\exp (\log (\Gamma)+\Re)$ is a closed subgroup of $N$. Arguing as in $\S 3$, we see that it is sufficient to work with the pair $(\Re / \Re, \Gamma \exp (\Re) / \exp (\Re))$ and the linear functional on $\mathfrak{R} / \mathfrak{R}$ defined by $\varphi$. In other words: it is no loss of generality to assume $\Re=0$.

We thus assume that we are dealing with the case $\Re=0$. In particular, we now know that $\mathfrak{R}$ has a one-dimensional center 3 on which $\varphi$ does not vanish. Let $\mathrm{B}_{1}=\{x \in \mathfrak{N}:[x, \mathfrak{N}] \subseteq \mathbb{Z}\}$. Then $\log (\Gamma) \cap \mathrm{B}_{1}$ we contain a basis for $\mathrm{B}_{1}$ (as is clear from the corresponding fact for the center). Let $y_{1}$ be an element of such a basis, and assume that $y_{1} \notin 8$, also.

Let $\mathfrak{N}_{1}=\left\{x \in \mathfrak{N}:\left[x, y_{1}\right]=0\right\} . \mathfrak{N}_{1}$ is readily seen to be an ideal in $\mathfrak{N}$ of codimension one. Let $\Gamma_{1}=N_{1} \cap \Gamma$, where $N_{1}=\exp \left(\mathfrak{R}_{1}\right)$. Clearly $\Gamma_{1}$ is a lattice subgroup of $N_{1}$, and the restriction $\theta$ of $\varphi$ to $\log \left(\Gamma_{1}\right)$ is integral. Hence we may assume, by 
induction, that we have already computed a direct-sum decomposition $K(\theta)$ $=\sum \bigoplus_{i=1}^{k} J_{i}$ of the subspace $K(\theta)$ in $L^{2}\left(N_{1} / \Gamma_{1}\right)$ into irreducible, invariant subspaces (for $U^{\Gamma_{1}}$ ). Let $U^{i}$ be the restriction of $U^{\Gamma_{1}}$ to $J_{i}$.

We now make use of the crucial point in Kirillov's proof of the theorem of his that we have cited: $I_{N}(\varphi)$ is the unitary representation of $N$ induced (in the sense of Mackey) by $U^{i}$. Hence we can realize $I_{N}(\varphi)$ as the representation $T^{i}$ of $N$ on $L^{2}\left(R, J_{i}\right)$ given by

$$
\left(T_{m x}^{i} f\right)(t)=U_{t[m]}^{i}\left(f\left(\chi_{0}+t\right)\right)
$$

which is to be interpreted as follows:

Because $N / N_{1}=R$, the extension

$$
1 \rightarrow N_{1} \rightarrow N \rightarrow N / N_{1} \rightarrow 1
$$

is split. Also, $\log (\Gamma) / \log \left(\Gamma_{1}\right)=Z$. Hence $\log (\Gamma)=\log \left(\Gamma_{1}\right) \oplus Z x_{1}$ for some $x_{1} \in \log (\Gamma)$, and clearly then, $N$ is the semidirect product $N_{1} \cdot X$, where $X=$ $\exp \left(\boldsymbol{R} x_{1}\right)$. In the equation (*) above, $m$ is any element of $N_{1}, \chi$ is the element $\exp \left(\chi_{0} x_{1}\right)$ of $X$ and $t \in R$. Finally, by $t[m]$, we mean the element

$$
\exp \left(t x_{1}\right) m \exp \left(-t x_{1}\right)
$$

of $N_{1}$.

Define $V^{i}: L^{2}\left(R, J_{i}\right) \rightarrow L^{2}(N / \Gamma)$ by

$$
\left(V^{i} f\right)(\Gamma m \chi)=\sum_{n \in Z}\left(f\left(\chi_{0}+n\right)\right)\left(\Gamma_{1} n[m]\right),
$$

where $m \in N_{1}$ and $\chi=\exp \left(\chi_{0} x_{1}\right)$. To see that $V^{i} f$ is well defined is easy:

Let $\gamma \in \Gamma_{1} ; \eta \in \exp \left(Z x_{1}\right)$. Then

$$
\begin{aligned}
\left(V^{i} f\right)(\gamma \eta m \chi) & =\left(V^{i} f\right)\left(\gamma\left(\eta_{0}[m]\right) \eta x\right) \\
& =\sum_{n}\left(f\left(\chi_{0}+\eta_{0}+n\right)\right)\left(\Gamma_{1} \gamma\left(\eta_{0}+n\right)[m]\right) \\
& =\sum_{n}\left(f\left(\chi_{0}+n\right)\right)\left(\Gamma_{1} n[m]\right) \\
& =\left(V^{i} f\right)(m \chi) .
\end{aligned}
$$

Finally, we must compute $V^{i} T_{m x}^{i}$ :

$$
\begin{aligned}
\left(V^{i} T_{m_{x}}^{i} f\right)(\Gamma p \eta) & =\sum_{n}\left[\left(T_{m x}^{i} f\right)\left(\eta_{0}+n\right)\right]\left(\Gamma_{1}(n[\grave{p}])\right) \\
& =\sum_{n} U_{\left(\eta_{0}+n\right)[m]}^{i}\left(\left[f\left(\chi_{0}+n+\eta_{0}\right)\right]\left(\Gamma_{1}(n[p])\right)\right) \\
& =\sum_{n}\left[f\left(\chi_{0}+n+\eta_{0}\right)\right]\left(\Gamma_{1}(n[p m])\left(\eta_{0}[m]\right)\right) \\
& =\left(V^{i} f\right)(\Gamma p \eta m \chi) \\
& =\left(U_{m x}^{\Gamma} V^{i} f\right)(\Gamma p \eta)
\end{aligned}
$$

Hence $V^{i} T_{m x}^{i}=U_{m x}^{\Gamma} V^{i}$. 
The proof is now complete, except to show that when $i \neq j, V^{i}\left(L^{2}\left(R, J_{i}\right)\right)$ $\perp V^{j}\left(L^{2}\left(R, J_{j}\right)\right)$. The latter is evident from the fact that $m \mapsto n[m]$ is a Haarmeasure preserving automorphism of $N_{1}$.

We now have a map $V: \boldsymbol{K}(\theta) \rightarrow \boldsymbol{K}(\varphi)$. This map is into and not onto, in general. In order to get the remainder of $\boldsymbol{K}(\varphi)$, we use the process described above, but with $\varphi \circ \operatorname{Ad}(\chi)$ in place of $\varphi$, where $\chi=\exp \left( \pm x_{1}\right), \exp \left( \pm 2 x_{1}\right), \ldots, \exp \left( \pm n x_{1}\right), \ldots$

\section{REFERENCES}

1. L. Auslander, On a problem of Philip Hall, Ann. of Math. (2) 86 (1967), 112-116. MR 36 \#1540.

2. L. Auslander et al., Flows on homogeneous spaces, Ann. of Math. Studies, No. 53, Princeton Univ. Press, Princeton, N. J., 1963, Chapter V. MR 29 \#4841.

3. A. A. Kirillov, Unitary representations of nilpotent Lie groups, Uspehi Mat. Nauk 17 (1962), no. 4(106), 57-110. (Russian) MR 25 \#5396.

4. C. C. Moore, Decomposition of unitary representations defined by discrete subgroups of nilpotent groups, Ann. of Math. (2) 82 (1965), 146-182. MR 31 \#5928.

5. J. Scheuneman, Two-step nilpotent Lie algebras, J. Algebra 7 (1967), 152-159. MR 36 \#225.

6. A. Weil, Sur certains groupes d'opérateurs unitaires, Acta Math. 111 (1964), 143-211. MR 29 \#2324.

INSTITUTE FOR ADVANCED STUdY,

Princeton, New Jersey 08540

UNIVERSITY OF MINNESOTA,

MinneAPOLIS, MinNeSOTA 55455 